\title{
Proteins in amorphous saccharide matrices: Structural and dynamical insights on bioprotection ${ }^{\star}$
}

\author{
S. Giuffrida ${ }^{1, a}$, G. Cottone ${ }^{1}$, G. Bellavia ${ }^{2}$, and L. Cordone ${ }^{1}$ \\ 1 Dipartimento di Fisica e Chimica, Università degli Studi di Palermo, Via Archirafi 36, I-90123, Palermo, Italy \\ 2 Unité Materiaux et Transformations UMR CNRS 8207, Université Lille 1, UFR de Physique Bât P5, 59655 Villeneuve d'Ascq, \\ France
}

Received 15 November 2012 and Received in final form 21 March 2013

Published online: 17 July 2013 - (C) EDP Sciences / Società Italiana di Fisica / Springer-Verlag 2013

\begin{abstract}
Bioprotection by sugars, and in particular trehalose peculiarity, is a relevant topic due to the implications in several fields. The underlying mechanisms are not yet clearly elucidated, and remain the focus of current investigations. Here we revisit data obtained at our lab on binary sugar/water and ternary protein/sugar/water systems, in wide ranges of water content and temperature, in the light of the current literature. The data here discussed come from complementary techniques (Infrared Spectroscopy, Molecular Dynamics simulations, Small Angle X-ray Scattering and Calorimetry), which provided a consistent description of the bioprotection by sugars from the atomistic to the macroscopic level. We present a picture, which suggests that protein bioprotection can be explained in terms of a strong coupling of the biomolecule surface to the matrix via extended hydrogen-bond networks, whose properties are defined by all components of the systems, and are strongly dependent on water content. Furthermore, the data show how carbohydrates having similar hydrogen-bonding capabilities exhibit different efficiency in preserving biostructures.
\end{abstract}

\section{Introduction}

Biopreservation is a relevant topic, in particular for its implications in food industry, pharmaceutics and medicine; large efforts are currently addressed to understand the mechanisms regulating the biopreservation processes, both in vivo and in vitro. In particular, amorphous saccharide matrices have been proven very efficient in protecting biostructures against adverse conditions such as drought or extreme temperatures [1].

Among sugars, trehalose $(\alpha, \alpha-\mathrm{D}, \mathrm{D}$-glucopyranosylglucopyranoside), a non-reducing disaccharide found in organisms able to overcome adverse environmental conditions, results the best stabilizer of biostructures [2]. The mechanism of bioprotection by trehalose is not yet fully elucidated and remains the focus of many researches. Its action has been related to the higher value of its glass transition temperature in comparison with other disaccharides [3]; to specific interactions between sugars and biological systems in the dry state (water replacement hypothesis [4]), or among sugars, protein and residual water at the interface preserving the native solvation (water entrapment or sugar preferential exclusion) $[5,6]$. It has

\footnotetext{
* Contribution to the Topical Issue "Neutron Biological Physics" edited by Giovanna Fragneto and Frank Gabel.

a e-mail: giuffrid@fisica.unipa.it
}

also been proposed that the high viscosity of sugar matrices could be at the basis of the reduction of large-scale internal protein motions leading to loss of structure and denaturation [7]. The above hypotheses are not mutually exclusive. The formation of a glassy state does not imply hydrogen bonding, as evidenced by measurements on dextran [8]. The efficiency of trehalose may be due to its ability in forming glassy structures in a wide hydration range, along with the hydrogen bond capability. However, this is not consistent with results on raffinose, which is less effective than other sugars [9], although it has a glass transition temperature comparable with that of trehalose, along with larger hydrogen bonding potential [10].

An approach based on structural studies of trehalosewater binary systems, proposes that the polymorphism of trehalose both in the crystalline and amorphous states is at the basis of its effectiveness [11]. The slow formation of dihydrate trehalose crystals, by water evaporation at high temperature and low moisture, would keep water molecules in the same hydrogen bond (HB) network as in the solvated trehalose, capturing the residual water molecules without disrupting the native structure of the biological systems. Further slow dehydration would produce anhydrous trehalose. The biostructures would therefore be protected by the inhibition of large-scale motions, preserving its active conformations. This process has bioprotective effects because of the existence of reversible 
paths among the different states, avoiding at the same time water crystallisation.

The very strong trehalose-water interaction may play a role in determining trehalose effectiveness. Raman and neutron scattering experiments $[12,13]$ on binary systems revealed the disruption of the tetrahedral network of water molecules on addition of trehalose. The consequent reordering of water molecules around the saccharide impairs ice formation and improves preservation.

Raman scattering experiments were performed also on protein-sugar solutions [14-19]. By comparing trehalose, sucrose and maltose systems, it was found out how trehalose is the most effective in stabilizing the folded secondary structure of the protein [14]. The experiments were carried out both in the low-frequency range and in the amide I band region, both providing a microscopic description of the process of thermal denaturation. In particular, amide I band is very sensitive to the unfolding of the secondary structure, whereas the low-frequency range provides significant information on protein dynamics and on coupling of protein and hydration-water dynamics. By comparing protein-trehalose solutions at different composition $[14,15,18,19]$, it has been confirmed that the main effect of trehalose is related to its capabilities to perturb the tetrahedral organization of water molecules and to strengthen the intermolecular $\mathrm{O}-\mathrm{H}$ interactions responsible for the stability of tertiary structure [17]. In this way, the thermal stability of the HB network of water contributes to the stabilization of the native tertiary structure and inhibits the first stage of denaturation, that is, the transformation of the tertiary structure into a highly flexible state with intact secondary structure. Trehalose was also observed to be the best stabilizer of the folded secondary structure, in the transient tertiary structure, leading to a high-temperature shift of the unfolding process (i.e. the second stage of denaturation) [14].

Proteins embedded in saccharide amorphous matrices at very high sugar concentration have been so far extensively studied by Neutron Scattering, Mössbauer spectroscopy, Flash Photolysis, Molecular Dynamics (MD) simulations [20-24]. At high sugar concentration, the dynamics of a protein was found to be highly inhibited, the inhibition markedly depending on the traces of residual water [22]. In particular, MD simulations of proteincontaining systems suggested that the protein surface is directly connected to an HB network including trehalose and water, in which, upon dehydration, the fraction of water molecules bridging protein and sugar increases, while the total amount of bridging water reduces. In this condition also the average number of hydrogen bonds, in which each water molecule is involved, was found to increase. These observations confirmed that the water replacement and water entrapment hypotheses are not mutually exclusive; this was also pointed out by recent MD simulations showing that trehalose molecules assemble in distinctive clusters on the surface of the protein; the flexibility of the protein backbone is then reduced due to the presence of these sugar patches [25].

The protein-matrix interaction, and its dependence on hydration, has been also studied by Infrared Spec- troscopy (FTIR) on carboxy-myoglobin (MbCO) embedded in trehalose matrices at different water content [2628 . The properties of the protein have been studied following the thermal evolution of the CO stretching band (COB, $\sim 1900-2000 \mathrm{~cm}^{-1}$ ), which is split into three different sub-bands (A substates) [29], each corresponding to a specific different environment experienced by the bound $\mathrm{CO}$ within the heme pocket [30]. The thermal behaviour of this band therefore gave information on the thermal interconversion among taxonomic and lower hierarchy substates, which can be evidenced, for example, by the thermal line broadening and peak frequency shifts. The properties of the matrix in the same systems have been studied following the thermal evolution of the adjacent Water Association band (WAB, 2000-2400 $\mathrm{cm}^{-1}$ ). This band is attributed to a combination of the bending mode of water molecules with intermolecular vibrational modes involving either other water molecules or non-water HBforming groups [26,31]. Due to its intermolecular origin, the thermal evolution of this band was assumed to reflect the thermal rearrangements of the water-containing matrix. A rough quantitative estimate of the thermal evolution of both the protein and the matrix was obtained measuring the functional distance of the spectra, at various temperature, from the respective spectrum measured at a reference temperature (Spectra Distance, SD). This is defined as $[26,32]$

$$
\mathrm{SD}=\sqrt{\sum\left(A(\nu, T)-A\left(\nu, T_{\mathrm{ref}}\right)\right)^{2} \Delta \nu},
$$

where $A(\nu)$ is the normalized absorbance at the frequency $\nu$ and $\Delta \nu$ is the frequency resolution. The normalization, performed by dividing by the total area under each curve, was needed to avoid that small thermal variation of the band population could alter the value of SD, which is meant to measure the change in band shape. The above quantity represents the deviation of the spectra at temperature $T$ from the spectrum in condition of minimal occurrence of thermal motions, which often corresponds to the lowest temperature investigated. This quantity was proposed to reflect the thermally induced readjustments of either the matrix $\left(\mathrm{SD}_{\mathrm{WAB}}\right)$ or the protein structure as evidenced by the bound $\mathrm{CO}$ molecule $\left(\mathrm{SD}_{\mathrm{CO}}\right)$. Considering that such structural changes imply protein internal motions, $\mathrm{SD}_{\mathrm{CO}}$ also conveys information on internal protein dynamics.

Overall, FTIR and MD results suggested that the rigidity of the HB network increases on drying. This network is mainly responsible for coupling the internal dynamics of the protein to that of the low-water matrix [28]. Saccharides protect biomolecules not by simply preserving their native solvation, but rather by locking their surface either directly or through networks of water molecules, thus hindering internal motions. Indeed, the formation of strong, extended HB networks, as in glassy ternary systems, sizably increases the energy penalty associated with solvent re-arrangements, needed for large-scale protein internal motions [34]. This was supported by vibrational echo experiments performed on different hemeproteins in trehalose glasses [35]. 
The formation of extended HB networks has been rationalized by Terahertz Spectroscopy, a useful tool for probing the collective modes of the water network. In particular, it has been reported that each solute affects in a peculiar way the fast collective network motions of the solvent, well beyond the first solvation layer. The total $\mathrm{THz}$ absorption would depend on both saccharide concentration and number of HB formed with water [36]. This suggested that, under suitable solutes concentration, largescale collective modes involving wide regions of the sample can set up, such long-range coherence failing if solute particles reduce their participation in HB networks.

In this respect, light scattering spectra on aqueous solutions of trehalose, recorded in a very wide frequency range $(0.6-36000 \mathrm{GHz})$ [37], indicate the presence of two separate solvent relaxation processes: a slow one, related to the solute dynamics, ascribed to hydrating water, and a fast one ascribed to bulk solvent. These results were confirmed by MD simulations [38] and in presence of protein [39].

The above reported data are in line with the peculiar effectiveness of trehalose with respect to other saccharides. However, we remind the reader that a lot of sugars do have bioprotective properties: actually different organisms can use different saccharides as bioprotectant (e.g. glucose in frogs). In the framework of various hypotheses for biopreservation, this is to be expected because saccharides have a rather homogeneous chemical structure, and all have a large hydrogen bonding capability, hence the ability to strongly influence HB networks.

This similarity in saccharide structure gives flattened effects both in solution and rubbery states, where viscosity and solvent dynamics dominate, and the embedded biomolecules face an "average solvent". When the entire system is in the glassy state, a very effective suppression of biomolecules dynamics is present, which in this case largely relies on specific solute-protein-water interaction. In the latter condition effects due to the different saccharide chemical structure are enhanced. Consequently, a deep understanding of the trehalose peculiarity has been achieved by comparing different saccharides, paying attention to the simultaneous exploration of the thermodynamic states of the entire systems, and of the transition between rubbery and glassy states. This was done by studying systems with different sugar/water ratios and at different temperatures.

In this work we review data obtained by our group on myoglobin embedded in disaccharide matrices (maltose, sucrose, trehalose). Studies on monosaccharides [40] pointed out that they cannot form hard glassy matrixes, which are the most effective toward bioprotection, so we will not discuss them.

As our model to rationalize the trehalose peculiarity relies on the characteristics of the HB network present in the system, we focus our review on the comparison of ternary (protein-saccharide-water) system with corresponding binary systems (saccharide-water). This shed light on the molecular basis of the complex relation among sugar, water and embedded proteins. Data from different and complementary techniques (MD, FTIR, Small An-

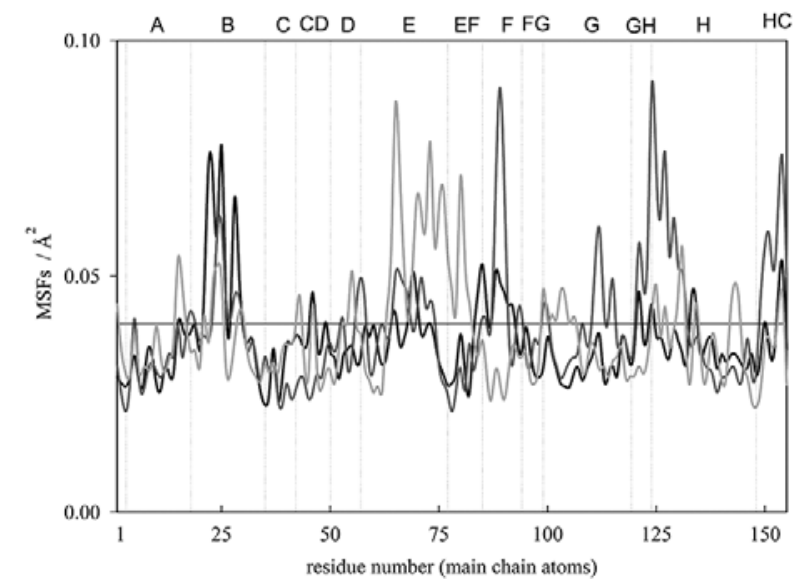

Fig. 1. Atomic mean square fluctuations as a function of residue number, averaged over the protein main chain atoms $\left(\mathrm{C}, \mathrm{C}_{\alpha}, \mathrm{N}\right)$. Data are calculated by averaging over 15 time blocks of $200 \mathrm{ps}$ each $[43,44]$. MbCO in $89 \% \mathrm{w} / \mathrm{w}$ trehalosewater (black line); $\mathrm{MbCO}$ in $89 \% \mathrm{w} / \mathrm{w}$ sucrose-water (darkgrey line); $\mathrm{MbCO}$ in $89 \% \mathrm{w} / \mathrm{w}$ maltose-water (grey line). Horizontal line stands for the average value. The labels on the top of the plot indicate secondary structure elements in MbCO.

gle X-Ray Scattering, SAXS, and Differential Scanning Calorimetry, DSC) are here reviewed and compared, with the aim to obtain a consistent picture of the bioprotection mechanisms with a multiscale approach, from the atomistic to the macroscopic level.

\section{Molecular dynamics}

To investigate at a molecular level the effects of homologue disaccharides on the protein structure/dynamics and the protein-solvent coupling, MD simulations were performed on $\mathrm{MbCO}$ embedded in disaccharide-water box (trehalose, sucrose, maltose) at $89 \% \mathrm{w} / \mathrm{w}(\sim 2.3$ water/sugar $)$ [41-43]. This concentration was chosen because it corresponds to the equilibrium water content of crystalline trehalose $(2$ water molecules per trehalose molecule), and it is also roughly comparable to the water content found in dry MbCO-trehalose matrices at environment temperature and pressure [26]. Protein atomic fluctuations, proteinsolvent $\mathrm{HB}$ at the protein interface, and solvent-solvent HB were analysed.

The atomic Mean Square Fluctuations (MSFs) along the protein chain in the three saccharides, calculated on hundreds of picoseconds time scale, are shown in fig. 1. The value averaged over the protein's main chain atoms were found similar for $\mathrm{MbCO}$ in trehalose, sucrose, and maltose, being $\sim 0.04 \AA^{2}$ at room temperature in each system. Data in fig. 1 show that MbCO experiences stronger and more uniform constraint from the external matrix in trehalose than in sucrose or maltose; this in turn agrees with the higher bioprotective efficiency of trehalose. A detailed comparison of atomic fluctuations points out that, in sucrose and maltose, residues with significant flexibility are still present, located at the $\mathrm{E}, \mathrm{F}$, and $\mathrm{H}$ helices as 
well as at the EF and GH loops. It is worth noting that these regions are those more involved in thermal denaturation, as reported by a MD study which compares the atomic fluctuations of the fully hydrated protein at 300 and $400 \mathrm{~K}[43]$.

MD simulations of a very concentrated liquid solution, namely MbCO embedded in a 50\% w/w (19 water/sugar) at $300 \mathrm{~K}$ [41], showed instead that the internal protein dynamics is unaffected by the presence of trehalose. Indeed, the value of the MSFs averaged on all the protein atoms was found to be $0.24 \AA^{2}$, in good correspondence with the values of $0.23 \AA^{2}$ [45] or $0.26 \AA^{2}$ [46] found in MD simulations of $\mathrm{MbCO}$ in a water solution. Similar results were obtained from simulations of lysozyme in a trehalose aqueous solution $(18 \% \mathrm{w} / \mathrm{w}, 90$ water/trehalose): the calculated fluctuations for the $\mathrm{C}_{\alpha}$ atoms showed no significant difference in the presence or absence of trehalose, the corresponding averages over the protein residues being $1.02 \AA^{2}$ and $0.98 \AA^{2}$, respectively [47].

Vitkup et al. [45] performed a MD simulation of MbCO in water in which water molecules were kept at $180 \mathrm{~K}$, while the embedded protein was maintained at $300 \mathrm{~K}$. This mimicked a "high viscosity solvent" uniformly surrounding the protein, whose MSFs resulted of very low amplitude and uniform over the whole chain. The MSFs in trehalose, shown in fig. 1, are most similar to the ones reported by Vitkup et al. in the case of "cold solvent". It is also known $[48,49]$ that, in protein-water systems, water translational motions, which allow complete exchange of protein-bound water molecules by translational displacement, are necessary for large-scale fluctuations, e.g. interconversion among high tier substates and structural relaxations. These studies pointed out that reducing the dynamics of the solvent/matrix as a whole has a strong effect on the dynamics of the protein. For a binary system composed of trehalose and water, it has been possible to disentangle the effects of the two components by shutting down the dynamics of one of them. To this aim, simulations of $\mathrm{MbCO}$ have been performed in a trealosewater $89 \% \mathrm{w} / \mathrm{w}$ system with constraints imposed on the water molecules. This has been made either by blocking both the translational and the rotational motions of the molecules, or by blocking only translations, while allowing the water molecule to rotate (unpublished results, work in progress).

The mean square fluctuations of $\mathrm{C}_{\alpha}$ atoms on the ten nanoseconds time scale are shown in fig. 2. Results clearly establish the role of water dynamics on the protein internal dynamics, in protein/trehalose/water systems at low water content [22]. In particular, fig. 2 shows that hindering of water translational diffusion is the primary mechanism in the reduction of protein atomic fluctuations throughout the protein. The extent of the reduction is similar to that of a system at $200 \mathrm{~K}$ in which all water degrees of freedom are preserved (fig. 2, dotted line). The average of the MSFs along the protein chain is $\sim 0.06 \AA^{2}$, pointing out that inhibiting water translational displacement at room temperature corresponds to slow down the nanosecond protein dynamics to the one measured on the $100 \mathrm{ps}$ time scale (see fig. 1), even in a ternary system.

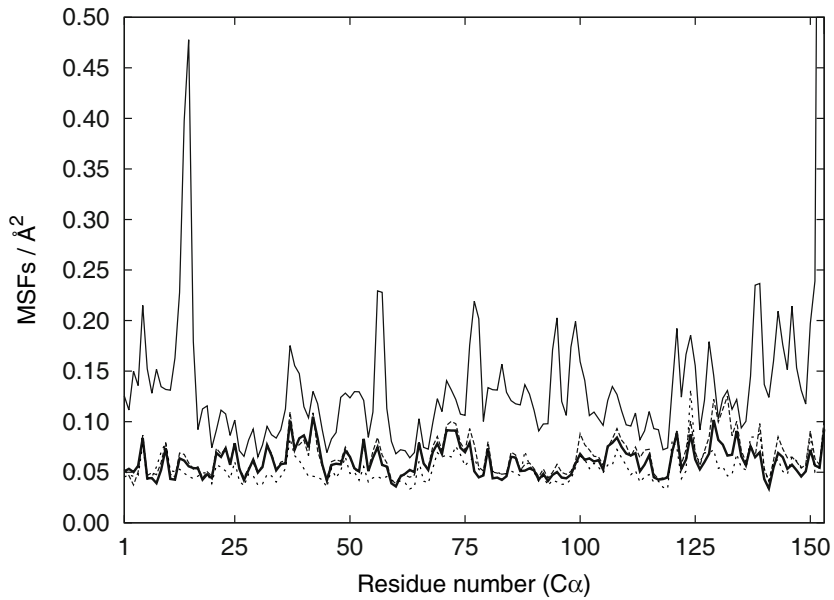

Fig. 2. Atomic mean square fluctuations at $300 \mathrm{~K}$ of $\mathrm{C}_{\alpha}$ atoms as a function of residue number, calculated over $10 \mathrm{~ns}$ trajectories. $\mathrm{MbCO}$ in $89 \% \mathrm{w} / \mathrm{w}$ trehalose-water at $300 \mathrm{~K}$ with frozen water molecules (both translation and rotation, thick black line); $\mathrm{MbCO}$ in $89 \% \mathrm{w} / \mathrm{w}$ trehalose-water at $300 \mathrm{~K}$ with frozen water molecules (only translation, dashed line); $\mathrm{MbCO}$ in $89 \% \mathrm{w} / \mathrm{w}$ trehalose-water at $300 \mathrm{~K}$ with no frozen atoms (thin black line); $\mathrm{MbCO}$ in $89 \% \mathrm{w} / \mathrm{w}$ trehalose-water at $200 \mathrm{~K}$ with no frozen atoms (dotted line).

MD was shown to be suitable to understand the effects of sugar matrices on the structure and dynamics of the protein, as it allows to determine the distribution of the mixed solvent at the macromolecule interface at the atomistic level. We calculated the ratio $N_{\text {OW }} /\left(N_{\text {OW }}+N_{\text {OS }}\right)$ for each sugar, where $N_{\text {OW }}$ and $N_{\text {OS }}$ indicate, respectively, the number of water oxygen atoms and sugar hydroxyl groups oxygen atoms found at distance $\mathbf{r}$ from the protein surface, irrespective of their binding state. The value of this ratio in the protein domain was reported to be sizably higher than the asymptotic value in each sugar system [43]. Accordingly, we suggested that, even in systems at $89 \% \mathrm{w} / \mathrm{w}$, water preferentially surrounds the protein. This is in agreement with the model proposed by Timasheff [6] for dilute sugar solutions or preferential hydration model, i.e., the solvent composition of the local protein domain is enriched in water relative to the average solvent composition. These results have been confirmed from experiments on $\mathrm{MbCO} /$ trehalose samples by Fayer and coworkers [35].

We also performed a Hydrogen Bond analysis on the simulated systems. We adopted the convention that a HB is present when the donor-acceptor distance is shorter than $3.5 \AA$ and the angle between the donor-acceptor axis and the donor-hydrogen bond is less than $30^{\circ}$ [50]. Results pointed out that the saccharide molecules are bound to protein mainly through only one out of the eight $\mathrm{OH}$ groups available for binding $(58 \%, 63 \%, 58 \%$ in trehalose, sucrose, and maltose, respectively). While the protein interacts either with single or multiple hydrogen bonds with water, the few sugar $\mathrm{OH}$ groups bound to the protein are in large majority linked through a single (donor) hydrogen bond. These results are in agreement with previous 


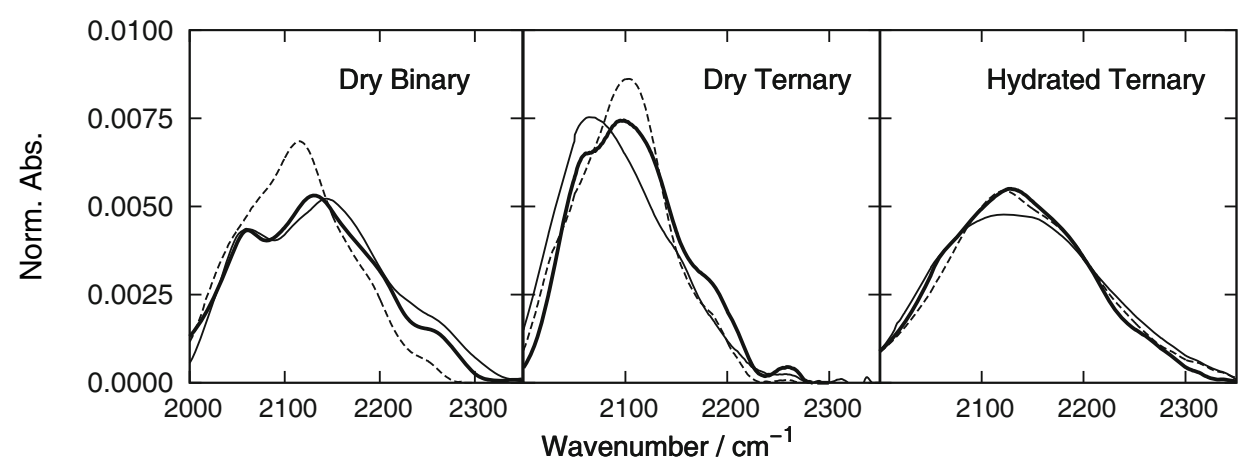

Fig. 3. Normalized spectra of the WAB at $300 \mathrm{~K}$ for binary dry samples (left panel), ternary dry samples (middle panel) and ternary hydrated samples (right panel), for the three studied saccharides: trehalose (thick lines) [26], sucrose (dashed lines) [27, 54], maltose (thin lines) [28].

experimental data [51], which suggested that the interaction of trehalose or sucrose with the protein is restricted to sites assumed to bind water molecules through single hydrogen bonds. It was also shown that the sugar-protein interaction does not affect the number of bound water molecules in the first hydration shell, which are likely to be tightly bound to the protein by multiple hydrogen bonds. Furthermore, along each simulated trajectory, the sugar molecules are found mainly bonding the carboxylate oxygen atoms of glutamate and aspartate residues as well as the peptide oxygen atoms, in agreement with IR data on trehalose-protein systems [8]. XAFS studies of cytochrome $\mathrm{c}$ in trehalose glasses at different content of residual water [52] pointed out a reduction of the mean square displacements of the central iron atom with respect to the first coordination shell and a distortion of the porphirin group. The authors suggested that the matrix induces conformational changes in the protein, which in turn result in structural distortions of heme group at the local atomic scale. Results from simulations showed that a trehalose, or a maltose, molecule is bound to heme propionate oxygen atoms in the $\sim 90 \%$ and $76 \%$ of the total number of configurations saved along the trajectories, respectively; this suggested that the heme could be involved in a direct interaction with the matrix. On the contrary, a sucrose molecule was found bound to heme propionate oxygen atoms only in the $15 \%$ of the total number of saved configurations.

To resume, MD simulations on the ns time scale provided a molecular picture of the effects of disaccharides on the protein internal dynamics, pointing out the key role of the residual water present in the systems on the local flexibility at residue level. Furthermore, analysis of the solvent composition at the protein surface showed a water excess with respect to the average bulk composition. This result was also strengthened by the hydrogen bond analysis, which, once more, pointed out the key role of water molecules in mediating the protein-matrix interaction.

\section{Infrared spectroscopy}

Saccharides are very hydrophilic molecules, able to bind several water molecules in their hydration spheres; this makes the properties of the saccharide matrices to strongly depend on water content, the evaluation, and control, of the hydration playing an important role in the study of these matrices. This evaluation can be made through various absolute techniques, such as e.g. gravimetry; however the study of the properties of the infrared bands of water, such as the WAB, conveys deeper information on the properties of water in these systems.

In fig. 3 the normalized profiles of the WAB for three disaccharides (trehalose, sucrose, maltose) are shown for binary and ternary systems dried under high vacuum at $80^{\circ} \mathrm{C}$, i.e. in their glassy state, and ternary samples hydrated at $60 \%$ relative moisture, i.e. in their rubbery state. As we are interested only in changes of the absorption profile, we report spectra normalized by dividing by the total area under the curve.

When comparing high and low hydration ternary samples a distinctive trend is present, irrespective of the saccharide: in the former (fig. 3, right panel) the band loses almost all its saccharide-specific features and approaches the shape it has in pure water [32]. This was suggested to arise both from the higher contribution of water-water interactions on the band and from a higher mobility of the water molecules, expected for a non-glassy system, resulting in an averaged absorption. WAB profiles in dry conditions (fig. 3, middle panel) are instead strongly dependent on the type of saccharide, as they arise from different pattern of population of HB local structures. In particular, in the three disaccharides here reported the band is divided in at least four components, whose frequency is roughly the same in all the samples, but whose population differs. As the WAB has been reported to originate from a water bending-libration combination, it has been possible to ascribe the high and low frequency components to water interacting with stronger and weaker HB, respectively. Although a detailed study of the components of the band has not yet been published, the comparison of the band shapes in different matrices, with and without embedded protein, provided data on the effects the presence of the protein plays on the matrix structure.

The comparison of the band profile in binary and ternary systems (fig. 3, left and middle panels) show that the studied sugars fall in two categories: in trehalose and 

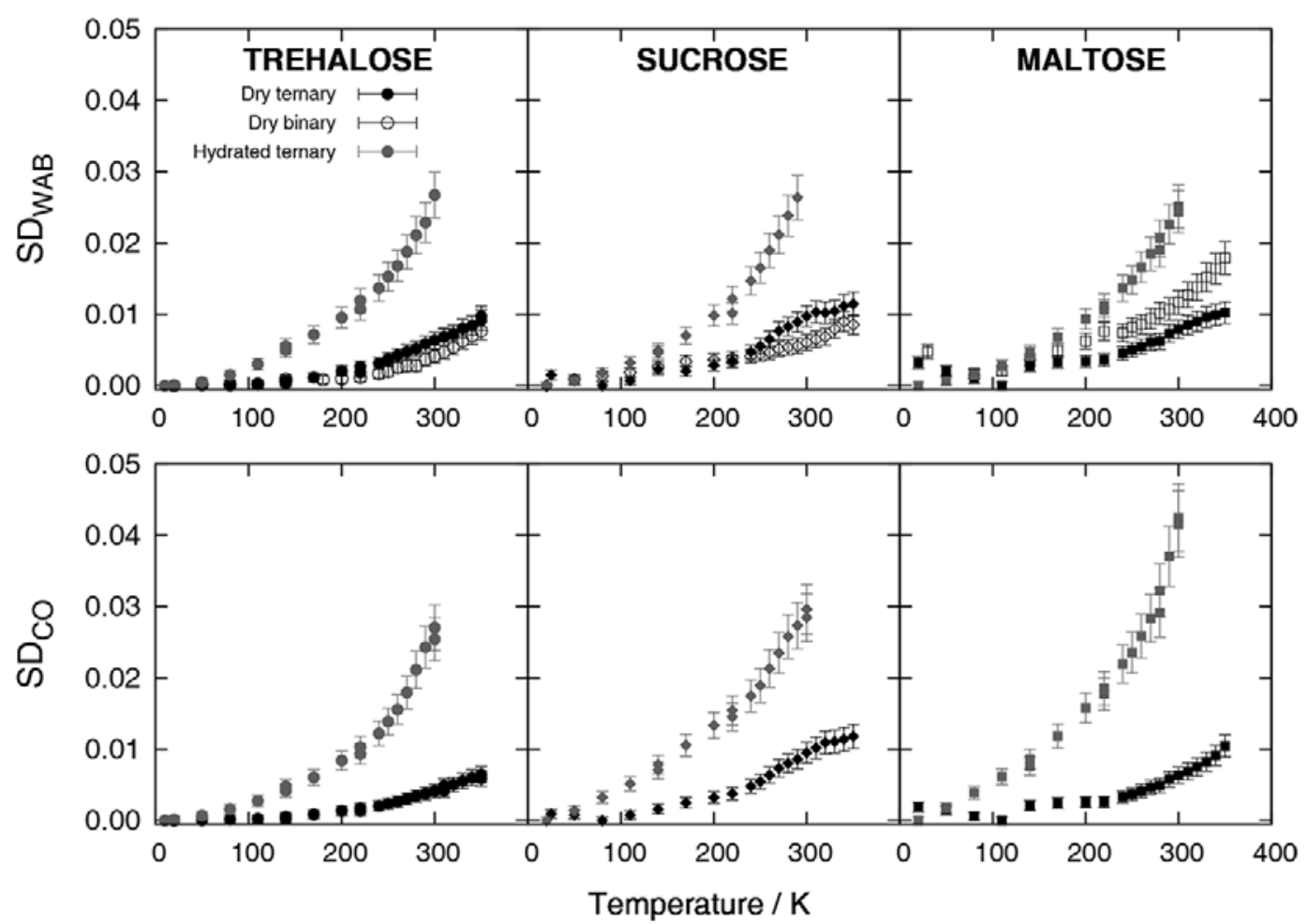

Fig. 4. Spectra distance, calculated for the $\mathrm{WAB}\left(\mathrm{SD}_{\mathrm{WAB}}\right.$, upper panels) and the $\mathrm{COB}$ ( $\mathrm{SD}_{\mathrm{CO}}$, lower panels). Data refer to ternary dry (black symbols), ternary hydrated (grey symbols) and binary (open symbols, only in the upper panels), for samples of trehalose (left panels), sucrose (middle panels), maltose (right panels) [28,32].

maltose (thick and thin continuous lines) the band shape undergoes a huge change, indicating that the introduction of protein alters strongly the distribution of HB structures; in sucrose (dashed lines) the band shape shows smaller changes. In particular, although the main component is always the central band at $\sim 2130 \mathrm{~cm}^{-1}$, which corresponds to bulk-like water absorption, in both maltose and trehalose one can notice an increase in the low-frequency components. The increase originates from the presence of weaker hydrogen bonding groups, as expected in case of protein-water or protein-sugar interaction. This is less evident in sucrose, indicating that the distribution of water molecules is not strongly altered. Hence the presence of the protein has only a weak influence in sucrose matrices; conversely the matrix does not change its HB networks to better host the protein. This can be at the basis of the reduced effectiveness of sucrose as bioprotectant.

In the maltose ternary system there is a larger population of the low-frequency component than in the trehalose one, indicating an average lesser strength of the $\mathrm{HB}$ network. Given that water-sugar hydrogen bonds are, in average, stronger than protein-water, this would indicate either that more water is present in the protein domain, or that protein is involved in a larger number of HB. This enables to put the three sugars in an order of HB network strength ( sucrose $>$ trehalose $>$ maltose), defined as the resistance to alteration upon protein adding. This order parallels the order of influence of these sugars on the homogeneity of the saccharide matrix on a larger scale as identified by SAXS (see sect. 4).
The concurrent study of the COB confirmed this behaviour, probing directly the protein alterations. We reported that a shoulder appears in the low-frequency side of the CO spectrum in sucrose matrices, which corresponds to a fourth A substate [27]. The occurrence of this fourth substate would arise from heme pocket structural modifications induced by sucrose-like units [42]. In addition, in this case the strong and scarcely alterable HB network in sucrose could be at the basis of the deformation of the protein, which is forced to adapt to this HB network.

The thermal alterations of both the matrix and the embedded protein were evaluated by using the SDs, calculated for the WAB ( $\left.\mathrm{SD}_{\mathrm{WAB}}\right)$ and of the $\mathrm{COB}\left(\mathrm{SD}_{\mathrm{CO}}\right)$ (shown in fig. 4). In dry samples both $\mathrm{SD}_{\mathrm{CO}}$ and $\mathrm{SD}_{\mathrm{WAB}}$ have very low values up to $\sim 200 \mathrm{~K}$ for all the sugars. This indicates lack of structural changes in the protein as well as in the matrix and implies that, within our sensitivity, only harmonic atomic motions are present in both. Above $200 \mathrm{~K}$ a very small increase is evident for both SDs, stemming from interconversion among very low tier substates.

The $\mathrm{SD}_{\mathrm{WAB}}$ for binary samples (fig. 4, empty points) has values comparable with dry ternary samples. In trehalose and sucrose, the thermal evolution of $\mathrm{SD}_{\mathrm{WAB}}$ is almost identical in the presence and in the absence of protein. Taking into account that the protein clearly affects the population of the components of the WAB (fig. 3), and that $\mathrm{SD}_{\mathrm{CO}}$ and $\mathrm{SD}_{\mathrm{WAB}}$ (both ternary and binary systems) have similar behaviour, it has been inferred the presence of a strict protein-matrix dynamical coupling. 
$\mathrm{SD}_{\mathrm{WAB}}$ for binary maltose is consistently above the value for ternary dry samples, and also above the value of the other binary systems. This result was explained by taking into account that the binary maltose matrix retains a large amount of water [32]. In the ternary sample, the high concentration of embedded protein might play a cohesive effect on the matrix, by allowing a strong water release, and therefore a reduction of the matrix dynamics. This agrees with the above data, confirming a major effect of protein on maltose with respect to other saccharides.

Hydrated ternary samples show higher values for both $\mathrm{SD}_{\mathrm{CO}}$ and $\mathrm{SD}_{\mathrm{WAB}}$ : the plots are rather linear up to $200 \mathrm{~K}$ and change slope above such temperature for all the sugars. The low temperature behaviour in $\mathrm{SD}_{\mathrm{CO}}$ indicates the hindrance of large scale dynamics of the protein, still allowing low tier conformational interconversions. The same in $\mathrm{SD}_{\mathrm{WAB}}$ points to an analogous hindrance of water motions at the protein-matrix interface. Above $200 \mathrm{~K}$, i.e. above the transition from a glassy to a rubbery state (see sect. 5 and fig. 6), the collapse of the HB network and the onset of translational displacement of protein-bound water enable protein large-scale motions (see also sect. 2) [26, $27,48]$. In this respect, the thermal behaviour of COB has been compared with the thermal behaviour of mean square displacements of protein hydrogen atoms as measured by Neutron Scattering experiments. It is worth noting that the onset of large scale interconversions, as detected by FTIR, almost coincides with the onset of protein nonharmonic motions as detected by Elastic Neutron Scattering [33].

In hydrated systems, the different $\mathrm{SD}_{\mathrm{WAB}}$ plots are roughly superimposable [28], confirming again the substantial similarity of the hydrated sugar-water matrices and of their thermal structural rearrangements. On the other hand, the different $\mathrm{SD}_{\mathrm{CO}}$ plots are not overlapping over the whole temperature range. This suggested differences in the heme pocket thermal dynamics with respect to the matrix dynamics, hence a weakening of dynamical coupling. This is in line with the different proteinwater-sugar structures at the interface observed by MD simulation in different sugar systems (see above and [42, 43]). MD data pointed out that the fraction of proteinbound water molecules, which is also bound to the sugar (or bridging water molecules), over the total number of protein-bound water, is higher in trehalose $(73 \%)$ than in sucrose $(66 \%)$ or maltose $(68 \%)$. This fraction of water molecules shared between protein and sugar could determine the strength of the constraints imposed by the matrix on the protein. Hence, this fraction could rationalize the larger local freedom for the protein internal motions in sucrose and maltose with respect to trehalose. The fraction of water molecules bridging different sugar molecules, irrespective of their interaction with the protein, was found to be $70 \%, 66 \%$, and $63 \%$ for trehalose, maltose, and sucrose, respectively; this suggested that trehalose is more able to form structures in which water and sugar molecules cross-connect through the whole system [26], i.e. even far from the protein surface. This result is in line with simulative and experimental data on binary mixtures, indicating that trehalose sizably modifies the hydrogen bond network and the water dynamics by tightly binding water molecules $[13,53]$.

To resume, FTIR spectroscopy has been exploited as a valid technique to explore, at one and the same time, properties of matrix and protein, by making use of suitable bands. This allowed to examine in detail the alterations of the HB network that establishes in the system. The temperature dependence of these bands has also been fruitfully exploited to obtain information on the substate interconversion in the protein and its relation with the matrix thermal evolution. With this technique it has been possible to point out the peculiarities of different disaccharides toward different aspects of the matrix-protein coupling.

\section{Small Angle X-ray Scattering: structural characterization of the host matrix}

The results from MD and FTIR enabled to study the relation between protein and matrix structure and dynamics at molecular level. However these techniques are not suitable to obtain information on larger structural alterations and in particular, because of their spatial scale, they are not able to mark the presence of micro- or mesoscopic inhomogeneities. We therefore performed SAXS measurements on binary (saccharide-water) and ternary (MbCOsaccharide-water) systems with the aim to detect the effects of the embedded protein on a larger spatial scale [54, 55]. SAXS was chosen since it is a most suitable technique to investigate micro-nano structures having electronic densities that differ from their surrounding.

Figure 5, left panel, shows the SAXS patterns of ternary samples compared with an example of a binary sample. The main feature of the binary samples is a $q^{-4}$ behaviour, which is already evident at the lower bound of the measured $q$ range in all the samples. This feature indicates the presence of domains of, at least, micrometric size and it is present in all the samples irrespective of the specific saccharide. The only difference is found in sucrose samples (data not shown), where these domains are present in a narrower $q$ range, indicating the superimposition of a number of smaller inhomogeneities stemming from the possible formation of nanocrystals. This is further confirmed by the occurrence of a small peak at $q \sim 0.15 \AA^{-1}$ in the pattern of binary sucrose sample. This effect could be expected by considering that sucrose samples have lower residual water content, with respect to the other sugars; this promotes crystallisation since sucrose crystallizes in anhydrous form [56].

In the case of protein-containing samples a signal, originating from the protein, is evident in the $0.1-0.4 \AA^{-1}$ $q$-range (see fig. 5). Myoglobin average radius resulted $\sim 14 \AA$ in all the sugar matrices studied, in agreement with the literature [57]. Because of the high protein concentration, interference among $\mathrm{Mb}$ molecules had to be taken into account, and data have been fitted with the Percus-Yevick function in decoupling approximation, with a spherical form factor for the protein [58]. This analysis, performed on the three disaccharides at the lowest temperature investigated, puts in evidence a peak corresponding 

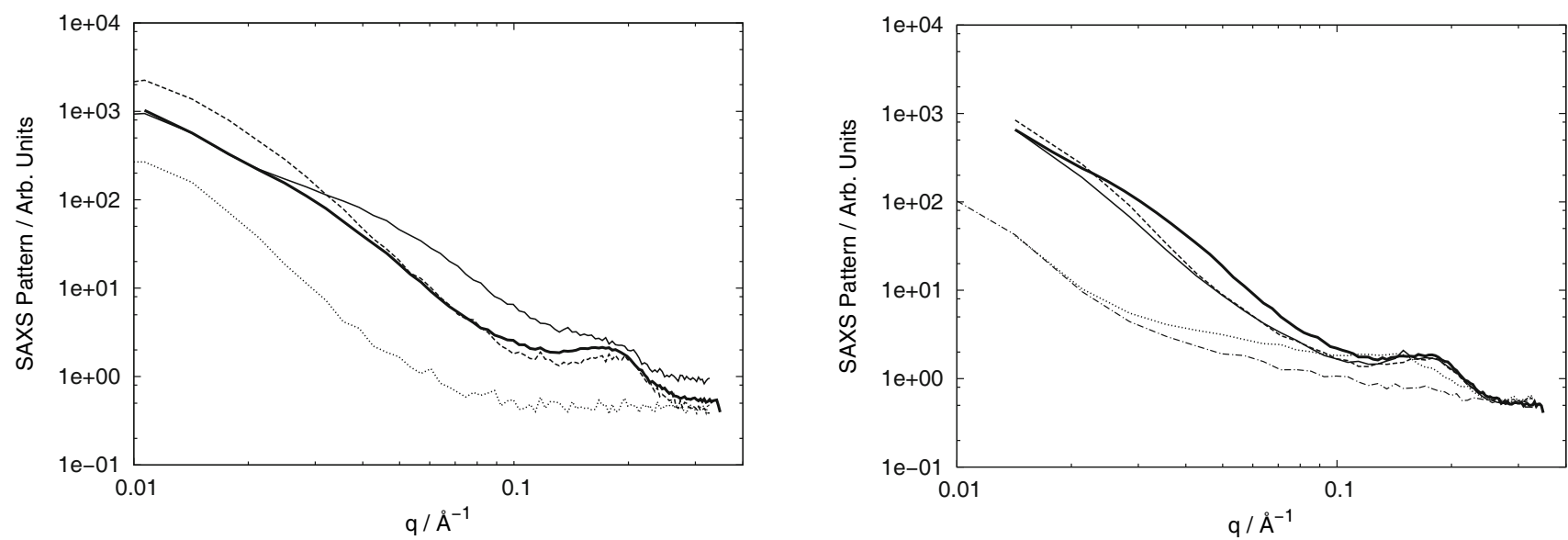

Fig. 5. SAXS experimental patterns. The details of sample preparation and experimental condition are reported in [54,55]. Left panel: trehalose-MbCO matrix (solid line), sucrose-MbCO matrix (thin line), maltose-MbCO matrix (dashed line), an example of binary (trehalose) matrix (dotted line). Right panel: trehalose-MbCO matrix at various hydrations: the water content is increasing along the order thick line (dry sample, as in the left panel), dashed line, thin line, dotted line, dot-dashed line.

to an average protein-protein distance of $\sim 31 \AA$, irrespective of the saccharide. This homogeneity of behaviour indicated that average distribution of myoglobin molecules is not significantly altered, hence no definite phase separation or aggregation occurs [55]. However, the volume fraction of interacting proteins was found different in the three sugars $(\eta=0.337,0.307$ and 0.24 , for trehalose, sucrose and maltose, respectively). As only proteins with similar shape and dimension can contribute to the interference peak, low $\eta$ values were reported to stand from the amount of denatured protein in the system, since $\eta$ values decrease with the amount of protein in native form. It has therefore been possible to establish an order of efficiency in preserving the protein based on the above $\eta$ values (trehalose $>$ sucrose $>$ maltose) [55]. The low efficiency of maltose was attributed to its reducing properties, which alter the protein structure and trigger intensive molecular cross-bridging.

It was also detected, in protein-containing samples, a feature within the $0.01-0.1 \AA^{-1}$ range, corresponding to large, broadly distributed structures. A fitting with the Debye function, assuming additivity, gave a distribution of domains whose size was strongly dependent on the specific saccharide, being $\sim 60 \AA$ in maltose, $\sim 120 \AA$ in trehalose, and $\sim 180 \AA$ in sucrose [55]. We attributed these domains to local differences in protein/sugar ratio. In particular, due to the high protein concentration in the samples, protein-rich regions constitute the SAXS background against which protein-poor domains are observable. These latter would be mainly constituted by sugar and water connected by extensive HB networks, stabilized by the partial seclusion of strong HB-maker saccharide from the weak HB-maker protein.

Upon hydration (see fig. 5, right panel) we noticed an increase in the size of these domains with increasing water content. This, in agreement with our model, could be rationalized by considering that the incoming water must preferentially concentrate at the more hydrophilic protein poor domains. Only very high hydration has an effect on the myoglobin signal, which broadens and shifts (fig. 5, right panel, dashed line). We attributed such behaviour to a change in the structure of the sample: the larger water availability makes the hydration of the protein increase and leads to the merging of the domains with the background (a sort of "melting"). Indeed, the new computed distance among protein molecules reaches $\sim 45 \AA$, which is compatible with the presence of at least one hydration layer surrounding the protein [54]. Upon further hydration (fig. 5, right panel, dot-dashed line) all correlation among proteins is lost, making the protein $S(q)$ peak disappear.

The nature of these domains resulted slightly different in the different saccharides. In particular, the MbCOmaltose system has the smallest inhomogeneities, whose average size is only twice the protein size. This agrees well with studies on analogous systems at molecular scale, with both simulative and experimental techniques, which supported the idea that maltose-water system are inhomogeneous due to maltose clustering $[28,59]$. The larger inhomogeneities present in MbCO-sucrose systems, along with the similar protein-protein average distance, led to suggest that less difference in protein concentration is present between the inhomogeneities and the background. This was also confirmed by the low scale factor for the Debye function obtained by fitting the data of sucrose samples: this indicates that either the inhomogeneities concentration is low or there is little contrast, as it would be expected if the difference in local protein concentration was small. Trehalose systems lay between these two extremes. The presence of a major perturbation of the system in trehalose or maltose ternary matrices well agrees with the FTIR results (see above, fig. 3, lower panels), which show that in trehalose and maltose, but not in sucrose, protein addition sizably alters the WAB shape.

The model inferred from SAXS data depicts, for ternary systems, the presence of protein-poor domains embedded in a protein-rich background. In the latter the protein is locked into its environment through a HB network involving saccharides and water, whereas the protein-poor 
domains are mainly constituted by saccharide-water regions, wherein few (or even no) myoglobin molecules are embedded. Unduly importance should not be credited to the specific nature of the saccharide. The main properties relying in the $\mathrm{HB}$ networks it forms, which could also be modulated by other components of the systems as well as iby different drying procedures. In this respect, it must be noticed that a similar inhomogeneous structure, obtained from Small Angle Neutron Scattering measurements, has been recently reported in frozen solutions of lysozyme, both containing and not containing saccharides [60], suggesting that the segregation is a general feature of these high concentrated protein solutions.

It has been reported $[11,61]$ that in dry binary trehalose systems, trehalose dihydrate regions may form by capture of water from the amorphous phase, which may act as source and sink of water. In line with this suggestion, we proposed that, in a similar way, the proteinpoor regions might play a buffering action against the daily variation of atmospheric humidity, useful in restarting the vegetative cycle in organisms in anhydrobiosis. A random distribution of the incoming water molecules might lead to a disordered onset of the function in the various compartments, which could eventually damage the organism. These domains would hence contrast the effects of moisture variations, by preferentially absorbing water molecules, and then slowly "melting" in a homogeneous phase above suitable hydration levels. This gentle transition would prevent a random onset of function and dynamics in different regions of the sample.

\section{DSC: thermodynamic characterization of the host matrix and protein denaturation}

According to Green and Angell [3] the efficiency in bioprotection could be related to the glassy transition temperature of sugar-water systems. Results so far described showed that the introduction of proteins in a sugar-water environment brings about significant effects on the system structure and dynamics.

With the aim of understanding how the presence of protein alters the properties of the glassy matrix, and how protein stability is related to the matrix glass transition temperature, DSC measurements were performed on binary disaccharide-water and ternary Mb-disaccharidewater systems, for non-reducing (trehalose, sucrose) and reducing (maltose) disaccharides.

Two $T_{g}$ regimes can be identified, in water-sugar systems, as a function of the water content (see fig. 6). In the low hydration range $T_{g}$ marks the transition between the liquid (rubbery) state and an homogeneous glassy state, and the $T_{g}$ behaviour can be described by the GordonTaylor equation. Otherwise, in the high hydration range $T_{g}$ marks the transition between an homogeneous liquid and an heterogeneous solid, composed by a mixture of ice and glass; in this region $T_{g}$ is constant because the excess water contributes only to ice fraction, while the glass composition remains constant. For all the disaccharides investigated, it has been possible to obtain homoge-

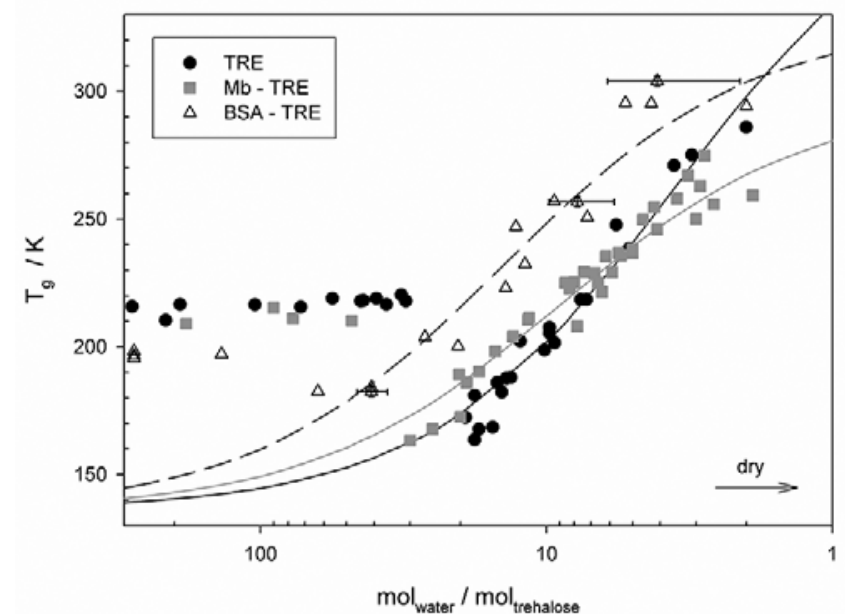

Fig. 6. $T_{g}$ as a function of hydration in ternary proteintrehalose-water systems in comparison with the correspondent binary trehalose-water system (full black circles): Mb (grey squares), BSA (open up-triangles). Lines are fittings in terms of the Gordon-Taylor equation [63,64]. As indicated by the arrow, hydration decreases from left to right.

neous glasses independent of the cooling rate above a definite critical water/sugar mole ratio. This value has been found to be 20, 18, and 15 water/sugar for trehalose, maltose, and sucrose, respectively. This order parallels the order reported for the perturbation of the HB network by sugars [12-14]. It is noteworthy that trehalose binary matrices exist as a homogenous glass in a wider hydration range than sucrose ones, wherein phase separation and crystallisation might occur even at relatively low water content, in agreement with results obtained by other techniques $[54,55]$.

Analysis of DSC data in the low water range showed that the presence of protein decreases the $T_{g}$ extrapolated at zero water content (see e.g. trehalose curves in fig. 6). This indicated that, under these conditions, looser water/disaccharide interactions are present: the protein, competing for water with disaccharide molecules, reduces the strength of the HB network encompassing the sample. This is in line with the mentioned simulative [41] and FTIR results [26].

A monotonic increase of the denaturation temperature $\left(T_{\mathrm{den}}\right)$ upon dehydration has been observed for nonreducing sugars (see e.g. the trehalose curve in fig. 7). The $T_{\text {den }}$ increase in maltose is very similar to the one in trehalose, however it looks monotonic only above 5 water/sugar mole ratio, whereas its behaviour loses regularity at lower hydrations, where the point apparently spread. This weird behaviour has been attributed to the occurrence of the Maillard reaction [62] which induces strong alterations of the thermodynamic properties of the whole system.

DSC measurements were also performed in trehalose matrices containing other proteins [64], namely lysozyme (LSZ), hemoglobin $(\mathrm{Hb})$ and Bovine Serum Albumin (BSA), at constant sugar/protein mole ratio, in order to investigate the size and charge effects (see e.g. BSA 


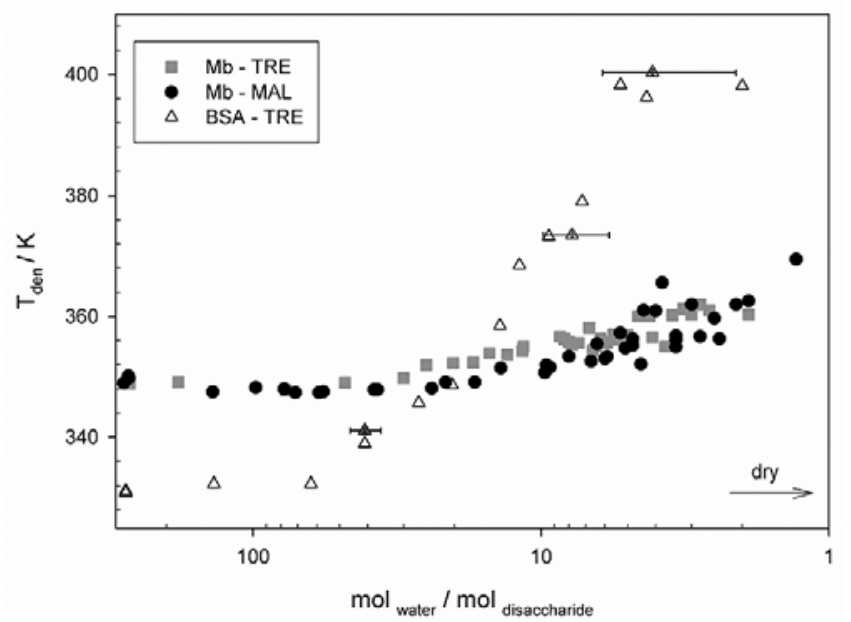

Fig. 7. $T_{\text {den }}$ as a function of hydration in ternary Mb-trehalose-water systems (grey squares), Mb-maltose-water (full black circles), BSA-trehalose-water systems (open up-triangles) $[63,64]$. As indicated by the arrow, hydration decreases from left to right.

curve in fig. 6). At high-to-intermediate hydration $(\geq 5$ water/sugar mole ratio), all ternary systems investigated showed the glass-liquid transition line shifted toward higher hydration, i.e. an increased $T_{g}$ at constant water/trehalose mole ratio. The effect is small for smaller proteins (Mb and LSZ) and large for larger proteins ( $\mathrm{Hb}$ and BSA), marking it as a size effect; at constant protein size, charge effects were found to play a minor role. In a trehalose matrix, the $T_{g}$ increase arises from a strengthening of the HB network, which, in turn, was attributed to the confinement experienced by the solvent. This was suggested, as expected, to be more severe in the case of larger than smaller proteins.

At very low hydration ( $<5$ water/sugar mole ratio), a $T_{g}$ decrease is observed independent on the protein size: this could be interpreted as the onset of sugar-protein competition for water, as above mentioned.

While for $T_{g}$ the effects of the use of different sugars or different proteins are of the same order of magnitude, $T_{\text {den }}$ is much more sensitive to the kind of protein (see fig. 7 ), as it is expected, considering that $T_{\text {den }}$ is a protein property. Moreover, the extent of protein stabilisation was found to depend also on charge effects. The $T_{\text {den }}$ increase is monotonous for all the proteins and scales in the order $\mathrm{Mb} \ll \mathrm{LSZ}<\mathrm{BSA} \cong \mathrm{HB}$, with values between $\sim 12 \mathrm{~K}$ for $\mathrm{Mb}$ and $\sim 70 \mathrm{~K}$ for the larger proteins, as could be seen comparing Mb-trehalose and BSA-trehalose curves in fig. 7. In the case of LSZ and BSA, the stabilizing effect for these proteins has been attributed to the strengthening of the matrix HB network, due to electrostatic interactions.

In all the systems $T_{\text {den }}$ and $T_{g}{ }^{1}$ was found to be linearly correlated (fig. 8): this has been proposed as a mark

\footnotetext{
1 The $T_{g}$ values considered in the correlation plot are only those referring to a homogeneous glass, i.e. where the GordonTaylor equation holds. When comparing different proteins a relative scale has been adopted: $\Delta T_{g}$ is referred to the highest
}
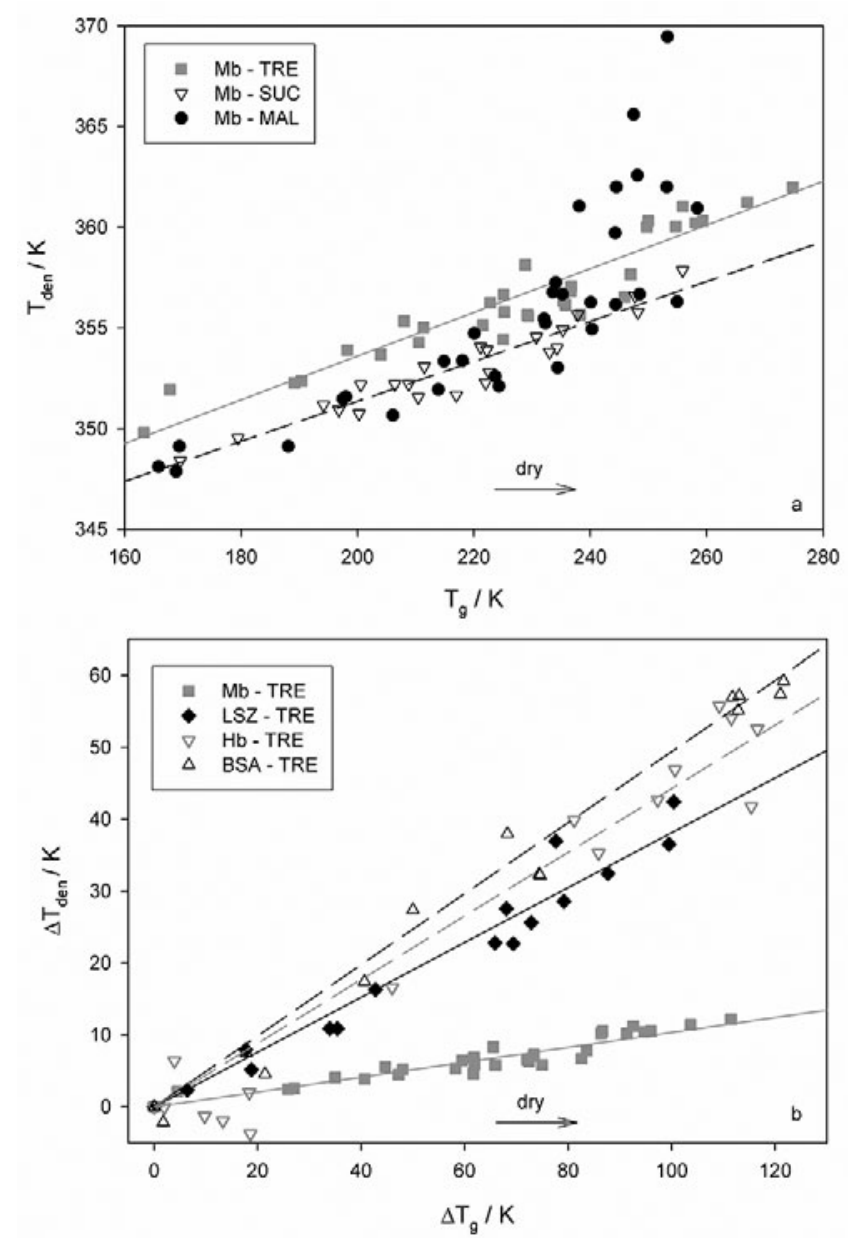

Fig. 8. Upper panel: $T_{\text {den }}$ as a function of $T_{g}$ for Mb-disaccharide-water systems: trehalose (grey squares), maltose (full black circles), sucrose (open down-triangles). Lower panel: $\Delta T_{\text {den }}$ as a function of $\Delta T_{g}$ for protein-trehalose-water systems: Mb (grey squares), LSZ (full black diamonds), BSA (open up-triangles) and $\mathrm{Hb}$ (open grey down-triangles) [63, 64]. Lines are linear fittings.

of the coupling between protein stability and matrix dynamics $[28,33,43]$. The collective properties that regulate the glass transition are linearly correlated to local properties involved in the protein denaturation, the stabilization extent depending on the specific protein-matrix interactions $[63,64]$. By comparing different sugars in $\mathrm{MbCO}$ ternary systems (fig. 8, upper panel), we noticed that the fitting line for trehalose lies above the sucrose one; that is, trehalose acts as a better stabilizer than sucrose at equal $T_{g}$. In this plot, the effects of Maillard reaction in maltose results in a strong deviation from linearity. Otherwise, the maltose values would be similar to sucrose.

By comparing different proteins (fig. 8, lower panel) different slopes have been observed, in the order $\mathrm{Mb} \ll$ LSZ $<\mathrm{Hb}<\mathrm{BSA}$. Although overwhelmed by confinement effects in determining $T_{g}$, the specific protein-matrix

water content to obtain a homogeneous system, and the corresponding value for $T_{\mathrm{den}}$ was taken as reference for $\Delta T_{\mathrm{den}}$. 
interactions do influence the denaturation process thus making the linear correlation specific for each protein.

Summing up, DSC has been utilised to complement the results obtained with the other techniques with a thermodynamics point of view. It has been possible to observe the different effects that disaccharides have on the denaturation properties of an embedded probe protein and, reciprocally, how different proteins shape the properties of the trehalose-protein glassy system. A linear dependence of $T_{\text {den }}$ as a function of $T_{g}$ was found in the whole range of hydration, where secondary processes (ice formation, Maillard reaction) do not take place. This relation allowed to show that trehalose better protects the protein, for a given $T_{g}$ value. A strong effect of the protein size was found on $T_{g}$ values, while both protein size and charge do affect $T_{\text {den }}$ for given $T_{g}$ values.

\section{Conclusions}

In this work we reviewed data from a complementary set of techniques, which enabled a description of the solid amorphous sugar matrices, of their mechanism of preservation and of the peculiarity of trehalose. FTIR spectra and MD simulations, at the atomistic level, SAXS measurements, on a supramolecular scale, and DSC, at thermodynamic level, depict a structure in which, in solid glasses, an extended water-saccharide HB network encompasses the whole system, whose rigidity is modulated by water content. The thermal behaviour of the embedded protein and of the matrix result coupled and both the components concur in defining the whole system properties. The comparison among various sugars matrices points out that, despite the structural similarity, saccharides behave differently in their interaction with the other components, leading also to different mesoscopic structures of the amorphous matrices.

The better biopreserving properties of trehalose can therefore be explained both in terms of stronger interactions both with water and the protein, leading to a stronger protein-matrix coupling, and in terms of a waterbuffering action played by the saccharide matrix, useful to prevent random onset of large biomolecular dynamics in different regions of the sample.

\section{References}

1. L.M. Crowe, Comp. Biochem. Physiol. A 132, 505 (2002).

2. J.H. Crowe, Adv. Exp. Med. Biol. 594, 143 (2007).

3. J. Green, C.A. Angell, J. Phys. Chem. 93, 2880 (1989).

4. J.F. Carpenter, J.H. Crowe, Biochemistry 28, 3916 (1989).

5. P.S. Belton, A.M. Gil, Biopolymers 34, 957 (1994).

6. S.N. Timasheff, Biochemistry 41, 13473 (2002).

7. J.G. Sampedro, S. Uribe, Mol. Cell Biochem. 256, 319 (2004).

8. S.D. Allison, B. Chang, T.W. Randolph, J.F. Carpenter, Arch. Biochem. Biophys. 365, 289 (1999).

9. W.F. Wolkers, H. Oldenhof, M. Alberda, F.A. Hoekstra, Biochim. Biophys. Acta-Gen. Subj. 1379, 83 (1998).
10. S.H. Gaffney, E. Haslam, T.H. Lilley, T.R.J. Ward, J. Chem. Soc. Faraday Trans. 84, 2545 (1998).

11. F. Sussich, C. Skopec, J. Brady, A. Cesàro, Carbohydr. Res. 334, 165 (2001).

12. C. Branca, S. Magazù, G. Maisano, P. Migliardo, J. Chem. Phys. 11, 281 (1999).

13. S. Magazù, G. Maisano, F. Migliardo, C. Mondelli, Biophys. J. 86, 3241 (2004).

14. A. Hédoux, J.-F. Willart, R. Ionov, F. Affouard Y. Guinet, L. Paccou, A. Lerbret, M. Descamps, J. Phys. Chem. B 110, 22886 (2006).

15. J.-A. Seo, A. Hédoux, Y. Guinet, L. Paccou, F. Affouard, A. Lerbret, M. Descamps, J. Phys. Chem. B 114, 6675 (2010).

16. A. Lerbret, F. Affouard, P. Bordat, A. Hedoux, Y. Guinet, M. Descamps, J. Chem. Phys. 24, 245103 (2009).

17. A. Hédoux, F. Affouard, M. Descamps, Y. Guinet, L. Paccou, J. Phys.: Condens. Matter 19, 205142 (2007).

18. A. Hédoux, J.F. Willart, L. Paccou, Y. Guinet, F. Affouard, A. Lerbret, M. Descamps, J. Phys. Chem. B 113, 6119 (2009)

19. A. Lerbret, F. Affouard, P. Bordat, A. Hédoux, Y. Guinet, M. Descamps, J. Non-Cryst. Solids 357, 695 (2011).

20. L. Cordone, M. Ferrand, E. Vitrano, G. Zaccai, Biophys. J. 76, 1043 (1999).

21. L. Cordone, G. Cottone, S. Giuffrida, G. Palazzo, G. Venturoli, C. Viappiani, BBA-Proteins Proteomics 1749, 252 (2005) and references therein.

22. L. Cordone, G. Cottone, S. Giuffrida, J. Phys.: Condens. Matter 19, 205110 (2007) and references therein.

23. A. Lerbret, F. Affouard, A. Hédoux, S. Krenzlin, J. Siepmann, M.C. Bellissent-Funel, M. Descamps, J. Phys. Chem. B 116, 11103 (2012).

24. F. Francia, M. Dezi, A. Mallardi, G. Palazzo, L. Cordone, G. Venturoli, J. Am. Chem. Soc. 130, 10240 (2008).

25. M.V. Fedorov, J.M. Goodman, D. Nerukh, S. Schumm, Phys. Chem. Chem. Phys. 13, 2294 (2011).

26. S. Giuffrida, G. Cottone, F. Librizzi, L. Cordone, J. Phys. Chem. B 107, 13211 (2003).

27. S. Giuffrida, G. Cottone, L. Cordone, J. Phys. Chem. B 108, 15415 (2004).

28. S. Giuffrida, G. Cottone, L. Cordone, Biophys. J. 91, 968 (2006).

29. H. Frauenfelder, F. Parak, R.D. Young, Annu. Rev. Biophys. Chem. 17, 451 (1988).

30. J. Vojtěchovský, K. Chu, J. Berendzen, R.M. Sweet, I. Schlichting, Biophys. J. 77, 2153 (1999).

31. D. Eisenberg, W. Kauzmann, The Structure and Properties of Water (Oxford University Press, London, 1969).

32. S. Giuffrida, G. Cottone, E. Vitrano, L. Cordone, J. NonCryst. Solids 357, 677 (2011).

33. L. Cordone, G. Cottone, S. Giuffrida, F. Librizzi, Chem. Phys. 345, 275 (2008).

34. U. Samuni, D. Dantsker, C.J. Roche, J.M. Friedman, GENE 398, 234 (2007) and references therein.

35. A.M. Massari, I.J. Finkelstein, B.L. McClain, A. Goj, X. Wen, K.L. Bren, R.F. Loring, M.D. Fayer, J. Am. Chem. Soc. 127, 14279 (2005).

36. M. Heyden, E. Bründermann, U. Heugen, G. Niehues, D.M. Leitner, M. Havenith, J. Am. Chem. Soc. 130, 5773 (2008).

37. M. Paolantoni, L. Comez, M.E. Gallina, P. Sassi, F. Scarponi, D. Fioretto, A. Morresi, J. Phys. Chem. B 113, 7874 (2009). 
38. L. Lupi, L. Comez, M. Paolantoni, D. Fioretto, B.M. Ladanyi, J. Phys. Chem. B 116, 7499 (2012).

39. S. Perticaroli, L. Comez, M. Paolantoni, P. Sassi, L. Lupi, D. Fioretto, A. Paciaroni, A. Morresi, J. Phys. Chem. B 114, $8262(2010)$.

40. D.P. Miller, J.J. de Pablo, J. Phys. Chem. B 104, 8876 (2000).

41. G. Cottone, G. Ciccotti, L. Cordone, J. Chem. Phys. 117, 9862 (2002).

42. G. Cottone, S. Giuffrida, G. Ciccotti, L. Cordone, Proteins 59, 291 (2005).

43. G. Cottone, J. Phys. Chem. B 111, 3563 (2007).

44. L. Maragliano, G. Cottone, L. Cordone, G. Ciccotti, Biophys. J. 86, 2765 (2004).

45. D. Vitkup, D. Ringe, G.A. Petsko, M. Karplus, Nat. Struct. Biol. 7, 34 (2000).

46. G. Cottone, L. Cordone, G. Ciccotti, Biophys. J. 80, 931 (2001).

47. R.D. Lins, C.S. Pereira, P.H. Hunemberger, Proteins 55, 177 (2004).

48. M. Tarek, D.J. Tobias, Phys. Rev. Lett. 88, 138101 (2002).

49. M. Tarek, D.J. Tobias, Biophys. J. 79, 3244 (2000).

50. A. Luzar, D. Chandler, Phys. Rev. Lett. 76, 928 (1996).

51. E.C. Lopez-Diez, S. Bone, Phys. Med. Biol. 45, 3577 (2000).
52. L. Giachini, F. Francia, L. Cordone, F. Boscherini, G. Venturoli, Biophys. J. 92, 1350 (2007).

53. P.B. Conrad, J.J. de Pablo, J. Phys. Chem. A 103, 4049 (1999).

54. A. Longo, S. Giuffrida, G. Cottone, L. Cordone, Phys. Chem. Chem. Phys. 12, 6852 (2010).

55. S. Giuffrida, M. Panzica, F.M. Giordano, A. Longo, Eur. Phys. J. E 34, 87 (2011).

56. G.M. Brown, H.A. Levy, Acta Cryst. B 29, 790 (1973).

57. K. Ibel, H.B. Stuhrmann, J. Mol. Biol. 93, 255 (1975).

58. J.S. Pedersen, Adv. Colloid Interface Sci. 70, 171 (1997).

59. A. Lerbret, P. Bordat, F. Affouard, M. Descamps, F. Migliardo, J. Phys. Chem. 109, 11046 (2005).

60. J.E. Curtis, H. Nanda, S. Khodadadi, M. Cicerone, H.J. Lee, A. McAuley, S. Krueger, J. Phys. Chem. B 116, 9653 (2012).

61. F. Sussich, C.E. Skopec, J.W. Brady, A. Cesàro, Food Chem. 122, 388 (2010).

62. F. Ledl, E. Schleicher, Angew. Chem., Int. Ed. 29, 565 (1990).

63. G. Bellavia, G. Cottone, S. Giuffrida, A. Cupane, L. Cordone, J. Phys. Chem. B 113, 11543 (2009).

64. G. Bellavia, S. Giuffrida, G. Cottone, A. Cupane, L. Cordone, J. Phys. Chem. B 115, 6340 (2011). 